\title{
The role of multimodal imaging in the diagnosis of an asymptomatic patient with congenital anomaly
}

\author{
Raluca Tomoaia ${ }^{1,2}$, Adrian Molnar ${ }^{1,2}$, Ruxandra Ștefana Beyer ${ }^{1}$, Alexandra Dădârlat-Pop ${ }^{1,2}$, \\ Florina Frîngư ${ }^{2,3}$, Diana Gurzău',3, Gelu Simư,3, Ioan Alexandru Minciună ${ }^{3}$, Bogdan \\ Caloian',3, Dumitru Zdrenghea ${ }^{2,3}$, Dana Pop ${ }^{2,3}$
}

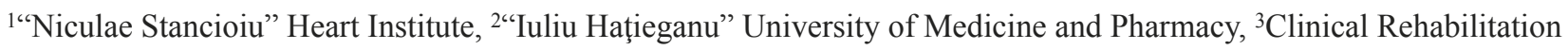
Hospital, Cardiology Department, Cluj-Napoca, România

\begin{abstract}
Anomalous left coronary artery from the pulmonary artery (ALCAPA) syndrome is a rare congenital coronary anomaly, which can cause potentially fatal complications, such as heart failure, myocardial infarction and sudden cardiac death. Only a few patients left untreated survive to adulthood. We highlight the importance of multimodal imaging in the diagnosis of ALCAPA syndrome in a young asymptomatic female patient with inducible ischemia on exercise. The patient was successfully treated with surgery.
\end{abstract}

Keywords: multimodal imaging; ALCAPA; coronary angiography; echocardiography

\section{Introduction}

Anomalous left coronary artery from the pulmonary artery (ALCAPA) syndrome is a rare congenital anomaly, affecting 1 of 300.000 newborns. Approximately $90 \%$ of patients die during the first year of life due to myocardial infarction and heart failure. The rest of the cases (18$25 \%$ ) develop sufficient myocardial collaterals between the anomalous left coronary artery and the normal right coronary artery and manage to survive to adulthood. This compensatory mechanism leads to a left to right shunt to the pulmonary artery, with coronary steal phenomenon, resulting in the development of myocardial ischemia, arrhythmias and heart failure later in life. Treatment of ALCAPA syndrome includes several surgical techniques,

Received 28.11.2019 Accepted 02.02.2020

Med Ultrason

2021, Vol. 23, No 2, 231-234

Corresponding author: Adrian Molnar MD, PhD

"Niculae Stancioiu" Heart Institute

19-21, Moților street,

400001, Cluj-Napoca, Romania

Phone: +40264597256

E-mail: adimolnar45@yahoo.com all associated with an increased morbidity [1]. We report the case of an asymptomatic 24-year-old female patient referred to our center for cardiology evaluation before joining the national basketball team. She was diagnosed with ALCAPA syndrome and was managed surgically with good outcome.

\section{Case report}

A 24-year-old previously asymptomatic female patient was referred to our cardiology department for evaluation before joining the national basketball team. She denied any history of palpitations, angina or dyspnea and had no family history of sudden cardiac death (SCD). There were no pathological findings on clinical examination. Electrocardiogram revealed diffuse ST-T changes. The exercise stress test showed further ST depression (up to 5 millimeters), which was predominant in V2-V6.

Routine transthoracic echocardiography (TTE) revealed multiple turbulent flows in the interventricular septum and in the lateral left ventricular (LV) wall, both in systole and diastole (fig 1A-D). There was also a significant thickening of the lateral LV wall, which was not dilated and had a preserved global systolic function (fig 1E), 


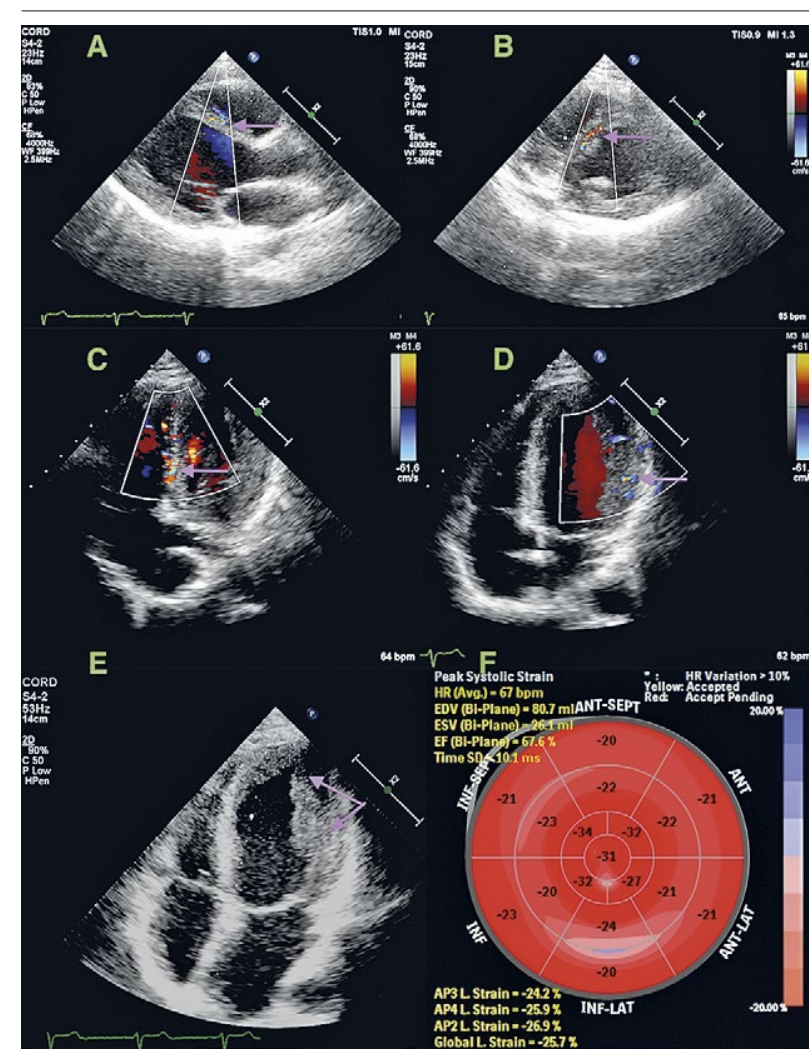

Fig 1. TTE showing multiple turbulent flows (arrows) in the interventricular septum from parasternal long (A) and short (B) axis. Same findings (arrows) from apical 4 chamber view in the interventricular septum (C) and in the lateral LV wall (D). Apical 4chamber view revealing significant thickening (arrows) of the lateral LV wall $(\mathrm{E})$. Bulls-eye showing reduced regional longitudinal strain at the level of the lateral wall (F).

but with reduced regional longitudinal strain at the level of the lateral wall (fig $1 \mathrm{~F}$ ). Therefore, the suspicion of multiple ventricular septal (VSD) defects was raised, possibly associated with left ventricular noncompactation (LVNC).

Magnetic resonance imaging (MRI)was afterwards performed, with visualization of a LV with preserved ejection fraction and sub-endocardial late gadolinium enhancement (LGE) at the level of the lateral and anterior septal LV walls. MRI criteria for LVNC were also positive (Petersen index of 4.4 at the level of the apex, 2.6 at the level of the lateral wall). Furthermore, there were multiple millimetric lodges within the myocardium, predominantly at the level of the same LV walls (fig 2). Thus, a coronary anomaly was suspected and a coronary computed tomography angiography (CCTA) was considered necessary as a next step.

CCTA revealed giant coronary arteries (CA) - the left CA (LCA) measured $10.3 \mathrm{~mm}$, the left anterior descending CA (LAD) $7.4 \mathrm{~mm}$ and the right CA (RCA) $8.8 \mathrm{~mm}$.
There was an anomalous origin of the LCA from the pulmonary artery (PA), with a normal origin of the RCA and many myocardial collaterals between the LAD and the RCA (fig 3).

Subsequently the patient underwent cardiac surgery with ligation of the proximal segment of the anomalous LCA and grafting of the left internal mammary artery into the distal segment of the artery. Two months later, the follow-up echocardiography revealed a significant reduction of the collateral vessels (fig 4). The patient decided to postpone joining the basketball team at that moment.

\section{Discussion}

ALCAPA syndrome is a very rare congenital disease and accounts for $0.25-0.5 \%$ of all congenital heart defects [1]. Survival is a rare finding in the adult population, depending on the development of inter-coronary collaterals between the left and the right CA. The left-toright shunt leads to coronary steal phenomenon, with low oxygenation of the lateral left ventricular (LV) wall as a consequence of preferential flow of the blood to the lowpressure PA as opposed to the myocardium. This mechanism may cause chronic myocardial ischemia and myocardial infarction, leading to malignant arrhythmias and SCD [2]. Once ALCAPA is diagnosed, surgery should be performed immediately [3].

Electrocardiography may raise the suspicion of this anomaly in a young adult, showing ischemic changes (most frequently negative $\mathrm{T}$ waves in DI and aVL) [4], which were also present in our patient.

Even though coronary artery angiography was the gold standard for the diagnosis of ALCAPA syndrome, noninvasive imaging is sufficient in the modern era [3]. Echocardiography may visualize a dilated RCA, retro-

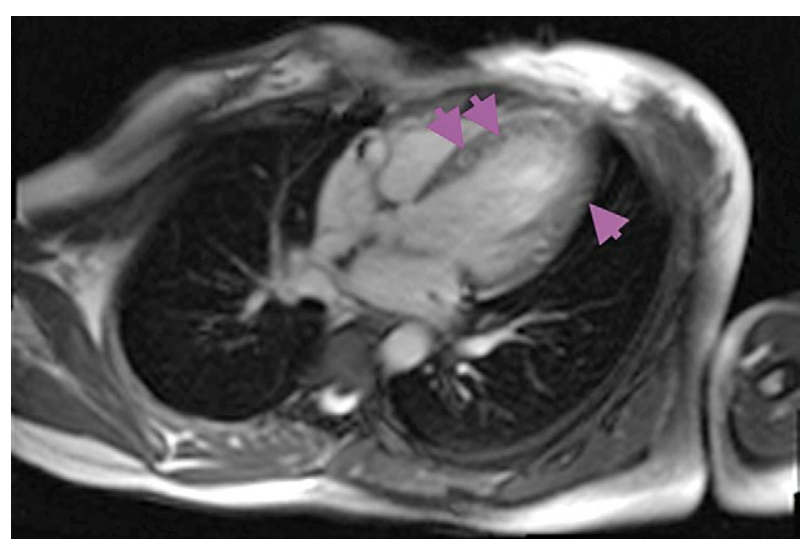

Fig 2. MRI visualizing multiple millimetric lodges within the myocardium, predominantly at the level of the septum and lateral LV wall (arrows). 


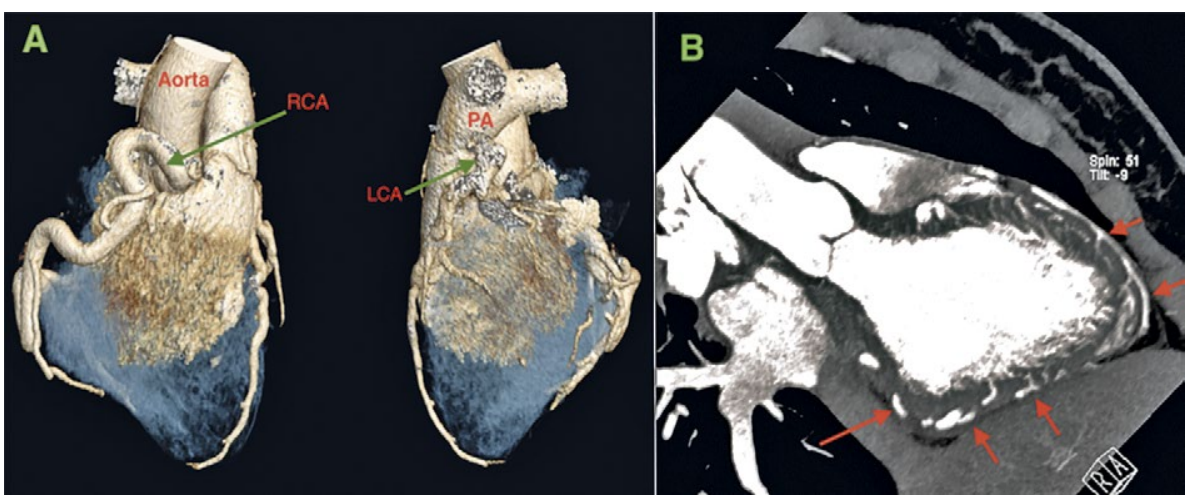

Fig 3. CCTA revealing an anomalous origin of the LCA from the PA with a normal origin of the RCA(A) and many collaterals (red arrows) between LAD and RCA (B).

grade flow from the LCA to the PA and the collaterals with systolic and diastolic blood flow [5]. Moreover, speckle tracking echocardiography reveals reduced longitudinal and circumferential strain in the regions corresponding to the LCA [2]. CCTA allows excellent spatial resolution to establish the origin and course of the $\mathrm{CA}$ [2]. MRI can be useful due to the benefit of LGE, which indicates fibrosis secondary to chronic ischemia [6]. Some of these findings were also present in our patient. There were septal and lateral color flow signals on echocardiography, with reduced regional strain at the level of the lateral LV wall. The CCTA revealed the abnormal course of the RCA with anastomoses between the right and left CA. MRI demonstrated fibrosis at the level of the lateral and anterior septal walls as a sign of chronic ischemia.

The connection between ALCAPA syndrome and LVNC can be explained by early embryonic development. ALCAPA is the result of abnormal septation of the conotruncus into the aorta and pulmonary artery or due to persistence of the pulmonary buds and involution of

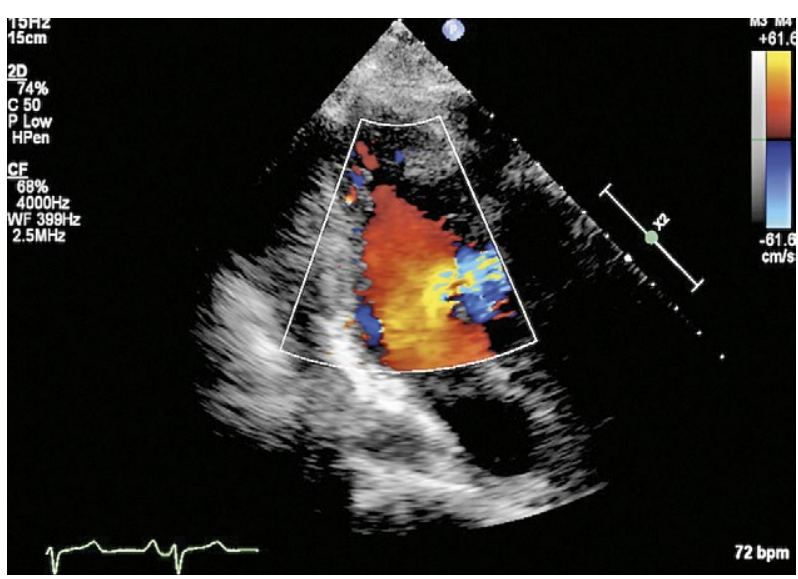

Fig 4. Three-months postoperative TEE showing significant reduction of the collateral vessels. the aortic buds. On the other hand, LVNC is caused by the arrest of the embryogenesis of the endocardium and myocardium, with coronary circulation being developed simultaneously during this process, when intratrabecular recesses are reduced to capillaries [2]. In our patient, both echocardiography and MRI showed a thickened lateral LV wall with positive criteria of LVNC, but with multiple milimetric lodges inside the myocardium, demonstrating the connection between the two processes (development of the CA and compactation of the LV).

If untreated, ALCAPA syndrome has a high mortality (80-90\%) [2]. SCD occurs mainly in young athletes and basketball players [1]. A literature review of 151 patients with this pathology found that $14 \%$ were asymptomatic and $62 \%$ of those with SCD were asymptomatic before the diagnosis was established [7].

The particularity of our case consists in the rarity of survival in patients with ALCAPA syndrome when left untreated, moreover with the patient being asymptomatic and physically active. Even though ALCAPA is rare in the adult population, the diagnosis is essential since early treatment may prevent myocardial damage. Current guidelines indicate that such patients may return to competitive sports 3 months postoperatively, provided that they remain asymptomatic and an exercise stress test does not show ischemia or important arrhythmias [3].

In conclusion we underline the importance of imaging in the early diagnosis of patients with ALCAPA syndrome. Furthermore, we highlight the connection between CA anomalies and LVNC, as these two processes coincide during early embryogenesis.

\section{References}

1. Harmon KG, Asif IM, Maleszewski JJ, et al. Incidence, cause, and comparative frequency of sudden cardiac death in national collegiate athletic association athletes: a decade in review. Circulation 2015;132:10-19. 
2. Elumalai G, Sujitha AS. "Anomalies origin of left coronary artery" its embryological basis and clinical significance. Elixir Embryology 2016;100:43446-43449.

3. Brothers JA, Frommelt MA, Jaquiss RDB, Myerburg RJ, Fraser CD, Tweddell JS. Expert Consesnsus guidelines: Anomalous aortic origin of a coronary artery. J Thorac Cardiovasc Surg 2017;153:1440-1457.

4. Dilawar M, Ahmad Z. Anomalous left coronary artery from pulmonary artery: Case series and brief review. OJPed 2012;2:77-81.

5. Patel SG, Frommelt MA, Frommelt PC, Kutty S, Cramer JW. Echocardiographic diagnosis, surgical treatment, and outcomes of anomalous left coronary artery from the pulmonary artery. J Am Soc Echocardiogr 2017;30:896903.

6. Boutsikou M, Shore D, Li W, et al. Anomalous left coronary artery from the pulmonary artery (ALCAPA) diagnosed in adulthood: Varied clinical presentation, therapeutic approach and outcome. Int J Cardiol 2018;261: 49-53.

7. Memon MKY, Amanullah M, Atiq M. Anomalous Left Coronary Artery from Pulmonary Artery: An Important Cause of Ischemic Mitral Regurgitation in Children. Cureus 2019;11:e4441. 